\title{
TRIM35 functions as a novel tumor suppressor in breast cancer by inducing cell apoptosis through ubiquitination of PDK1
}

\author{
Rong WANG*, Ke-Liang HUANG, Lian-Xiang XING \\ Department of Clinical Laboratory, Huangpu Branch, Shanghai Ninth People’s Hospital, Shanghai Jiao Tong University School of Medicine, \\ Huangpu, Shanghai, China \\ ${ }^{*}$ Correspondence: jyhfywr1968@163.com
}

Received August 23, 2021 / Accepted December 21, 2021

\begin{abstract}
Breast cancer is the most common cancer in women. Novel mechanisms and targets are urgently needed to understand and treat this disease due to the complexity of breast cancer. In this study, we evaluated the expression level of tripartite motif-containing (TRIM) 35 in various breast cancer cell lines by qPCR and immunoblot. Cell proliferation assay and flow cytometry were performed upon overexpression and depletion of TRIM35. Xenograft tumor model was applied to validate the findings observed in vitro. The correlation between TRIM35 and outcomes of breast cancer patients was investigated by analyzing The Cancer Genome Atlas database. We observed differential expression of TRIM35 in various breast cancer cell lines. Overexpression of TRIM35 significantly inhibited cell proliferation and promoted cell apoptosis. On the contrary, depletion of TRIM35 exerted the opposite effects on cell proliferation and apoptosis. Mechanistically, TRIM35 reduced PDK1 by ubiquitination, resulting in the degradation of PDK1. Overexpression of TRIM35 significantly suppressed ZR7530 cell line-derived xenograft tumor growth by inducing apoptosis. Finally, a lower level of TRIM35 was associated with a poor prognosis in patients. In conclusion, TRIM35 functions as a tumor suppressor to suppress breast cancer proliferation by inactivating AKT signaling through the increased ubiquitination of PDK1, resulting in the promotion of apoptosis.
\end{abstract}

Key words: TRIM35, AKT, PDK1, ubiquitination

Breast cancer (BC) is the most common cancer of women and the second leading cause of female cancer-related deaths. BC accounts for $10 \%$ of the newly diagnosed cancers every year worldwide [1]. Many risk factors, e.g., age, sex, breast disease history, family medical history, genetics, and exogenous hormone, affect the initiation and progression of $\mathrm{BC}$. The key principles for the treatment of $\mathrm{BC}$ are to diminish localized relapse and distant metastasis. So far, the therapeutic strategies contain surgery, hormone, chemotherapy, radiotherapy, and targeted therapy [2, 3]. Despite many therapeutic strategies were applied to treat BC, the 5-year survival of BC remains $22 \%$. Thus, it is important to provide individuated therapies for patients.

Many signaling pathways are engaged in the initiation and progression of $\mathrm{BC}$, including the TGF- $\beta /$ Smad pathway, MAPK pathway, Wnt/ $\beta$-catenin pathway, PI3K/AKT pathway, and NFkB pathway. It has been well documented that activation of the TGF- $\beta$ pathway promotes metastasis of $\mathrm{BC}$ via enhancing the epithelial to mesenchymal transition (EMT), resulting in the death of $\mathrm{BC}$. Inhibition of the TGF- $\beta$ pathway could restrain the metastasis of $\mathrm{BC}$, improve survival, and lower the relapse probability of BC patients [4]. It has been reported that the overexpression of epidermal growth factor receptor 2 (HER2) activates the Wnt/ $\beta$-catenin pathway, promoting EMT, migration, and invasion of BC cells $[5,6]$. In another study, overexpression of $N F \kappa B$ was obviously upregulated in invasive $\mathrm{BC}$ specimens compared with normal breast tissues [7], and AGEs could exacerbate triple-negative $\mathrm{BC}$ through active ERK and NFKB pathways [8]. In addition, inactivating the $\mathrm{PI} 3 \mathrm{~K} / \mathrm{AKT} / \mathrm{GSK}-3 \beta / \beta$-catenin pathway can suppress migration and invasion of $\mathrm{BC}$ cells $[9,10]$. Hepatocyte growth factor (HGF) activating PI3K/AKT and MAPK pathways increase the expression of cyclooxygenase 2 (COX2), resulting in the upregulation of Matrix metallopeptidase 9 (MMP9), and eventually induces migration and invasion of BC cells [11]. Introduction of inhibitor(s) of mechanistic target of rapamycin (mTOR) complex 1 (mTORC1) exerts therapeutic benefits for recurrent and metastatic HER2(+) BC [12]. Herein, targeting the PI3K/AKT/mTOR pathway potentiates new strategies to treat $\mathrm{BC}[13,14]$. 
Tripartite motif-containing protein 35 (TRIM35) is a member of the RING-B box-coiled-coil (RBCC) family, which has a highly conserved order consisting of a RING domain followed by one or two B-Box domains and then a coiled-coil domain. TRIM35, with the activity as an E3 ligase, could induce cell apoptosis [15]. Ubiquitin-proteasome pathway, one of the most important regulatory mechanisms, regulates physiologies of cells through specific degradation of proteins in cells [16]. It has been well documented that TRIM proteins, including TRIM35, TRIM21, and TRIM37, play roles in both tumor inhibition and progression. TRIM21 regulates the progression of glioblastomas by ubiquitin-mediated degradation of 6-phosphofructokinase, platelet type (PFKP) which is regulated by PTEN and PI3K/ AKT pathways [17]. TRIM37 could promote proliferation, migration, and invasion of glioblastoma through activating the PI3K/AKT pathway [18]. In contrast, TRIM35 was considered a tumor inhibitor to prevent tumor cell growth, colony formation, and tumor formation [19]. For example, overexpression of TRIM35 significantly restrained tumor cell proliferation and stimulated tumor cell apoptosis in hepatic cancer [20]. Moreover, TRIM35 prohibits the proliferation of hepatic cancer cells via reducing phosphorylation of pyruvate kinase muscle isozyme 2 (PKM2) [21]. However, the roles of TRIM35 in BC have not been fully understood. In this study, we explored the roles and potential mechanisms contributing to BC initiation and progression to seek possibilities for clinical applications.

\section{Patients and methods}

Patient samples. 27 pairs of $\mathrm{BC}$ and normal tissues were obtained from Shanghai Ninth People's Hospital with written informed consent. A human BC tissue chip was purchased from SHANGHAI OUTDO BIOTECH CO., LTD.

Bioinformatics analysis. TRIM35 expression levels of BC patients were analyzed using The Cancer Genome Atlas (TCGA) databases. Survival analysis used the data from GSE12276 [22]. Gene set enrichment analysis (GSEA) was performed by the JAVA program (http://www.broadinstitute. org/gsea) using the MSigDB portal. The enrichment analysis was performed and showed from high enrichment score to low enrichment score. The gene sets with a false discovery rate $(\mathrm{FDR})<0.25$ and $\mathrm{p}$-value $<0.05$ were considered as significantly enriched genes [23].

Cell lines and cell culture. ZR7530, MCF-7, T47D, SKBR3, BT474, and MCF10A cell lines were purchased from the Cell Bank of the Chinese Academy of Sciences (Shanghai, China). ZR7530 and SKBR3 cells were cultured in DMEM medium, MCF-7, T47D, and BT474 cells were cultured in RPMI-1640 medium, and MCF10A cells were cultured in DMEM/F12 medium. To make the complete growth medium, the following components were added to the medium: fetal bovine serum (Gibco, Carlsbad, CA, USA) to a final concentration of $10 \%, 100 \mathrm{U} / \mathrm{ml}$ penicillin, and $100 \mathrm{U} / \mathrm{ml}$ strepto- mycin (Gibco). All cells were cultured at humidified conditions with $5 \% \mathrm{CO}_{2}$ at $37^{\circ} \mathrm{C}$.

Real-time PCR assay. Total RNA was extracted using TRIzol reagent (Invitrogen, Carlsbad, CA, USA). M-MLV (Promega, Madison, WI, USA) kit was used to reverse transcribe RNAs $(2 \mu \mathrm{g})$ to cDNA. The relative expression levels of genes were quantified by real-time PCR with the SYBR Green qPCR Master Mix (Thermo Fisher Scientific Inc, Grand Island, NY, USA) on a 7500 PCR system (Applied Biosystems, Foster City, CA, USA). The $\beta$-actin was used as endogenous control and the $2^{-\Delta \mathrm{Ct}}$ or $2^{-\Delta \Delta \mathrm{Ct}}$ method was used to calculate the relative expression levels. The primers used were as follows: TRIM35 Primer F: 5'-ACCAGCTACCGCTTCTCGC-3', Primer R: 5'-GTGGGCAGTGTCCTTCACC-3'; PDK1 Primer F: 5'-GAGAGCCACTATGGAACACCA-3', Primer R: 5'-GGAGGTCTCAACACGAGGT-3'; $\beta$-actin Primer F: 5'-TGAACCCCAAGGCCAACC-3', Primer R: 5'-GGGCATACCCCTCGTAGATG-3'.

Western blot assay. Cells were harvested and lysed with RIPA buffer (Bio-Rad Laboratories, Inc., Hercules, CA, USA) containing a protease inhibitor cocktail (Sigma, St. Louis, MO, USA). A bicinchoninic acid assay kit (Thermo Fisher Scientific Inc.) was used to measure protein concentrations, allowing the same amounts of proteins to be separated by SDS-PAGE and transferred to PVDF membranes. Then, the membranes were blocked with $5 \%$ skim milk for $2 \mathrm{~h}$, incubated with primary antibodies and secondary antibodies. At the end, the bands were visualized using an enhanced chemiluminescence detection system (Bio-Rad Laboratories). The antibodies used were as follows: anti-TRIM35 (Ab186442), anti-XIAP (Ab229050), anti-PDK2 (Ab68164), anti-PDK1 (Ab202468), and anti- PI3K (Ab4255) were purchased from Abcam (St. Louis, MO, USA). Anti-AKT (\#9272), anti-p-AKT (\#4056), anti-Bad (\#9292), and anti- $\beta$-actin (\#4970) were purchased from Cell Signaling Technology (Danvers, MA, USA).

Lentivirus targeting TRIM35. Overexpression and knockdown lentiviruses targeting TRIM35 and PDK1 were purchased from Genechem company (Shanghai, China). The sequences for shRNA were as follows: shTRIM35-1: 5' - CCAACCACACCCTCAACAA-3'; shTRIM35-2: 5'-GGAGACAAGGCAGAAGCAA-3', shTRIM35-1: 5' - GGGCGAGCTGTCT T TCTAT-3'; shPDK1 - 1: 5' - TTTTCACAAGCATTCACTGAT-3'; shPDK1-2: 5' - AGTCATAAATAGCTTTAGCAT-3'; shPDK1-3: 5'-TCATCTTCACAGTCAAATCCT-3'; shNC: 5'-TTCTCCGAACGTGTCACGT-3’.

Cell proliferation analysis. A CCK-8 assay (Beyotime, Jiangsu, China) was performed to evaluate cell proliferation. Cells (3000 cells per well) were seeded into 96-well plates and cultured. At 24, 48, and $72 \mathrm{~h}, 10 \mu \mathrm{l}$ of CCK-8 and $90 \mu \mathrm{l}$ of cultured medium without fetal bovine serum was added to each well and further cultured for $1 \mathrm{~h}$. The absorbance was detected at an optical density of $450 \mathrm{~nm}$ using a Microplate Reader (Bio-Rad Laboratories). Five replicate wells were included for each group and each timepoint. 
Cell apoptosis analysis. An Annexin V and PI kit (BD Biosciences, San Jose, CA, USA) was used to analyze cell apoptosis. Cells were seeded in 6-well plates and transfected with relevant lentiviruses for another $48 \mathrm{~h}$. All the cells and culture medium were collected and suspended in $1 \times$ binding buffer supplied by the kit. Then, $5 \mu \mathrm{l}$ of Annexin V-FITC was added to the cells. After 10 min incubation in the dark, $5 \mu$ l of propidium iodide was added and incubated for $5 \mathrm{~min}$. Cell apoptosis was analyzed using flow cytometry (BD Biosciences).

Xenograft mouse model. The nude mice (6 weeks old) were purchased from Shanghai SLAC Laboratory Animal Co. Ltd. (Shanghai, China). All mice were kept with free access to food and water at $12 \mathrm{~h} \mathrm{light/dark} \mathrm{cycle.} \mathrm{All} \mathrm{the} \mathrm{animal} \mathrm{experi-}$ ments were approved by the Ethics Committee of Shanghai Ninth People's Hospital Shanghai Jiao Tong University. 12 mice were randomly divided into 2 groups: vector group and TRIM35 overexpression group. $5 \times 10^{6}$ ZR7530 cells transduced with vector or TRIM35 overexpressing lentiviruses were subcutaneously injected into the flank of the mice in the vector group and the TRIM35 overexpression group, respectively. The tumor size was measured every 3 days for 35 days, then mice were euthanized.

The hematoxylin and eosin stain (H\&E) staining and TUNEL assays. Tumor samples were fixed in $4 \%$ paraformaldehyde for $24 \mathrm{~h}$, embedded in paraffin, and cut into slices $(3 \mu \mathrm{m})$. Then, the slices were dewaxed, hydrated, and stained with hematoxylin and eosin. The images of each sample were obtained using an optical microscope (NIKON).

A TUNEL kit (Bio-Rad Laboratories, Inc) was used to detect apoptosis of tumor samples according to the manufacturer's instructions.

Co-immunoprecipitation (Co-IP). Cells were harvested and lysed with IP lysis buffer (Thermo Fisher Scientific Inc, Grand Island, NY, USA) containing a protease inhibitor cocktail (Roche). Supernatants of the lysates were incubated with protein G-Agarose immunoprecipitation reagent (Roche) together with the corresponding primary antibodies (anti-TRIM35, Abcam, Ab87169; anti-PDK1, Abcam, Ab207450) and incubated overnight at $4^{\circ} \mathrm{C}$. After three washes, the immunoprecipitates were boiled with SDS sample buffer, analyzed by immunoblotting.

Immunohistochemistry (IHC). The IHC analysis was carried out as described previously. The primary antibodies used were mouse anti-human TRIM35 (BIOSS, bs-9149R) and rabbit anti-human PDK1 (BIOSS, bsm-54037R). Immunoreactive scoring (IRS) method was scored for the expression. The PP was scored as follows: $0 \%, 0 ; 0-25 \%, 1$; 25-50\%, 2; 50-75\%, 3; and 75-100\%, 4. The SI was determined as follows: absent, 0 ; weak, 1 ; moderate, 2 ; and strong, 3; SI <3: negative-low expression, 3-9: high expression. IRS $=$ percentage of positive cells $(\mathrm{PP}) \times$ staining intensity $(\mathrm{SI})$.

Statistical analysis. Data were shown as mean \pm SD. Statistical analysis was performed with a two-tailed unpaired Student's t test between two groups, and a one-way ANOVA between multiple groups. A p-value $<0.05$ was considered significant.

\section{Results}

A lower level of TRIM35 correlates with a poor prognosis in BC. To investigate the effects of TRIM35 in $\mathrm{BC}$, we first evaluated the expression level of TRIM35 in the patients derived from TCGA database. As shown, a lower level of TRIM35 was observed in tumors than that in normal tissues (Figure 1A). Furthermore, we detected the TRIM35 mRNA level in 27 pairs BC and normal tissues and observed a lower level of TRIM35 in tumors compared with normal tissues (Figure 1B). Moreover, we analyzed the correlation between TRIM35 mRNA level and survival probability using the data from GSE12276 and found that a lower level of TRIM35 was associated with shorter overall survival than those with a higher level of TRIM35 (Figure 1C).

Based on the IHC staining results of BC tissue array, the samples were divided into high- and low-TRIM35 protein expression groups (Figure 1D). Furthermore, TRIM35 expression was found to be significantly $(\mathrm{p}<0.05)$ associated with several clinical parameters, including histologic grade, tumor size, AJCC stage, and tumor stage (Table 1). All these results indicated that TRIM35 played an important role in BC.

Table 1. Correlation between the TRIM35 expression and clinicopathological characteristics in BC patients.

\begin{tabular}{|c|c|c|c|c|}
\hline \multirow{2}{*}{ Characteristics } & \multirow{2}{*}{$\begin{array}{c}\text { No. Patients } \\
(\mathbf{n}=\mathbf{8 0})\end{array}$} & \multicolumn{2}{|c|}{ TRIM35 expression } & \multirow{2}{*}{ p-value } \\
\hline & & High No. (\%) & Low No. (\%) & \\
\hline Age (years) & & & & 0.4910 \\
\hline$>50$ & 30 & $14(46.7)$ & $16(53.3)$ & \\
\hline$<50$ & 50 & $28(56)$ & $22(44)$ & \\
\hline Histologic grade & & & & 0.0317 \\
\hline 1 & 23 & $11(47.8)$ & $12(52.2)$ & \\
\hline 2 & 44 & $28(63.6)$ & $16(36.4)$ & \\
\hline 3 & 13 & $3(23)$ & $10(77)$ & \\
\hline Tumor size $(\mathrm{cm})$ & & & & 0.0029 \\
\hline$\geq 2$ & 56 & $23(41.1)$ & $33(58.9)$ & \\
\hline$<2$ & 24 & $19(79.2)$ & $5(20.8)$ & \\
\hline AJCC stage & & & & 0.02 \\
\hline I & 20 & $13(65)$ & $7(35)$ & \\
\hline II & 46 & $28(60.8)$ & $18(39.2)$ & \\
\hline III & 14 & $3(21.4)$ & $11(78.6)$ & \\
\hline Tumor stage & & & & 0.0139 \\
\hline $\mathrm{T} 1$ & 22 & $10(45.5)$ & $12(54.5)$ & \\
\hline $\mathrm{T} 2$ & 45 & $30(66.7)$ & $15(33.3)$ & \\
\hline $\mathrm{T} 3$ & 13 & $3(23.1)$ & $10(76.9)$ & \\
\hline Distant metastasis & & & & 0.4557 \\
\hline Presence & 23 & $11(47.8)$ & $12(52.2)$ & \\
\hline Absence & 57 & $34(59.6)$ & $23(40.4)$ & \\
\hline
\end{tabular}

Note: Differences between groups were determined by the $\chi^{2}$-test. 
A

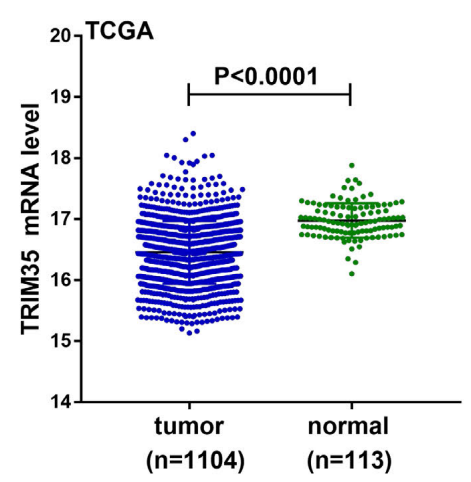

B

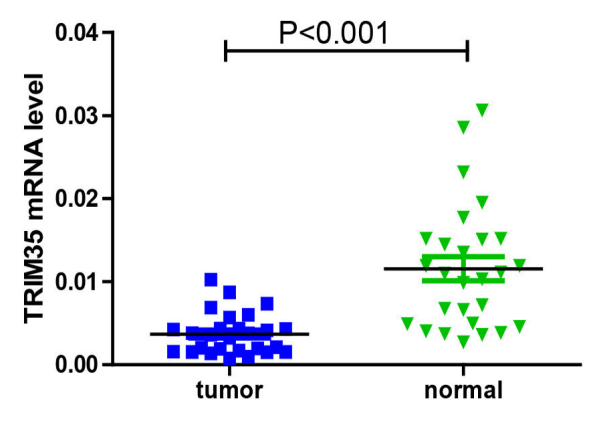

C

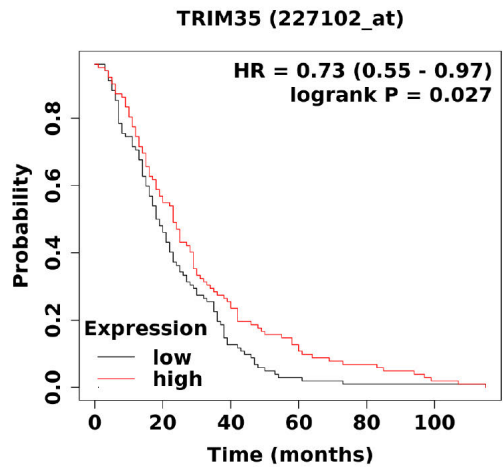

D

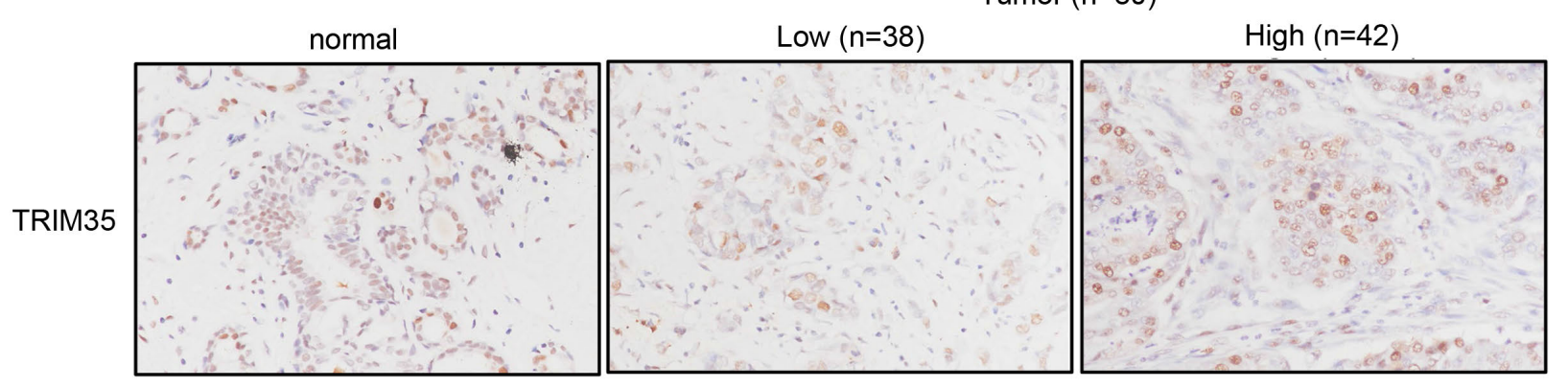

Figure 1. A low level of TRIM35 was associated with a poor prognosis of BC. A) TRIM35 expression level was analyzed using the data from TCGA. B) TRIM35 expression levels were examined in normal and BC tissues from 27 patients with BC. C) Kaplan-Meier analysis of overall survival rates of patients with BC from GSE12276 samples, based on TRIM35 expression levels. D) IHC against TRIM35 was performed in a BC tissue array (200×).

Overexpression of TRIM35 inhibits cell proliferation and induces cell apoptosis in $\mathrm{BC}$ cell lines. To investigate the roles of TRIM 35 in BC, we firstly evaluated the transcription levels of TRIM35 in various BC cell lines, including BT474, MCF-7, SKBR3, T47D, ZR7530, and mammary epithelial cell line MCF10A. As illustrated in Figure 2A, we observed a higher level of TRIM35 in MCF10A cells compared with $\mathrm{BC}$ cell lines. In BC cell lines, higher levels of TRIM35 were observed in SKBR3 cell line but the lower levels of TRIM35 in ZR7530 and MCF-7 cell lines. In agreement, we next examined the protein levels of TRIM35 in these cell lines and found similar results that the relative higher levels of TRIM35 in MCF10A and SKBR3 cell lines but lower levels of TRIM35 in MCF-7 cell line and barely disappearance of TRIM35 in the ZR7530 cell line (Figure 2B). Based on the expression level of TRIM35 in the various BC cell lines, we chose ZR7530 and MCF-7 cell lines for TRIM35 overexpressing and SKBR3 cell line for TRIM35 knockdown in the consequent studies due to the relatively lower and higher endogenous level of TRIM35, respectively. To study the roles of TRIM35 in BC behaviors, we overexpressed TRIM35 in ZR7530 and MCF-7 cell lines, then subjected them to cell proliferation assay by measuring the absorbance at OD 450 . As shown in Figure 2C, ectopic overexpression of TRIM35 significantly suppressed cell proliferation in both cell lines $(\mathrm{p}<0.0001)$. In agreement, ectopic overexpression of TRIM35 significantly induced apoptosis in the two cell lines as well (Figure 2D). Taken together, overexpression of TRIM35 suppresses BC cell proliferation but promotes $\mathrm{BC}$ cell apoptosis.

Depletion of TRIM35 promotes BC cell growth but prohibits cell apoptosis. To validate our findings in cells with TRIM35 overexpression, we then depleted TRIM35 in SKBR3 cells with a higher level of TRIM35. As illustrated in Figure 3A, TRIM35 was effectively depleted in SKBR3 cells via shRNAs transfection. Similarly, immunoblot assay also showed a significant reduction of TRIM35 after transfection of shRNAs against TRIM35 (Figure 3B). To further study the roles of TRIM35 in BC behaviors, we depleted TRIM35 in SKBR3 cell line, then subjected them to cell proliferation assay by measuring the absorbance at OD 450. As shown in Figure 3C, depletion of TRIM35 significantly stimulated cell proliferation in SKBR3 cell line $(\mathrm{p}<0.0001)$. In agreement, depletion of TRIM35 significantly prevented apoptosis in SKBR3 cell line as well (Figures 3D, 3E). Taken together, depletion of TRIM35 promotes BC cell proliferation but inhibits BC cell apoptosis.

Manipulation of TRIM35 induces cell apoptosis via inhibiting AKT signaling. Next, we sought to dissect the mechanisms contributing to the phenotypes observed in Figures 2 and 3. We performed a gene set enrichment assay (GSEA) in TCGA database and observed inactive AKT signaling signature enriched in patients with a higher level 



D


Annexin $\mathrm{V}$


Figure 2. Overexpression of TRIM35 inhibits cell proliferation and induces apoptosis. A) qPCR to examine expression levels of TRIM35 in various BC cell lines. B) Expression levels of TRIM35 in various BC cell lines were detected by immunoblot (IB). C) Cell proliferation assay in BC cells overexpressing TRIM35. D) Measurement of apoptosis in cells overexpressing TRIM35 by flow cytometry (upper). Quantification of flow cytometry (lower).

of TRIM35. Meanwhile, apoptosis signature was enriched in patients with a higher level of TRIM35 (Figure 4A). These data suggest that a high level of TRIM35 is correlated with the inactivation of the AKT pathway and induces apoptosis. To test this notion, we overexpressed TRIM35 in ZR7530 and
MCF-7 cell lines, followed by IB to detect AKT signaling and apoptosis. As shown in Figure 4B, overexpression of TRIM35 significantly reduced the level of phosphor AKT at S473 in ZR7530 and MCF-7 cell lines. In agreement, the elevation of Bad and reduction of XIAP were observed in ZR7530 and 
A



C

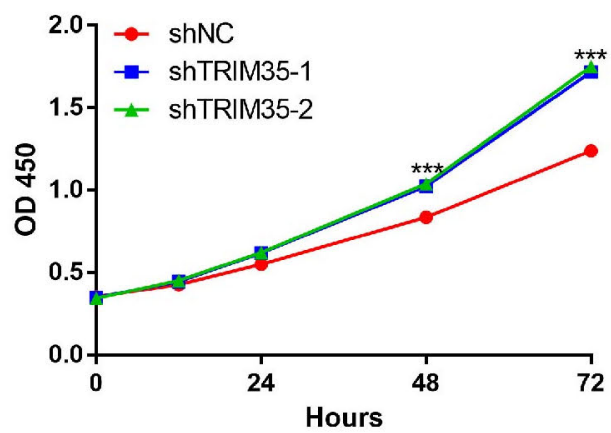

B

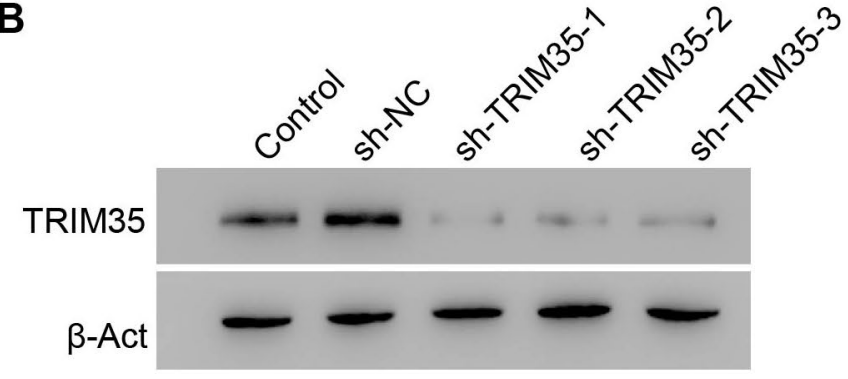

E



D


Annexin V

Figure 3. Depletion of TRIM35 promotes cell proliferation and inhibits apoptosis. A) qPCR to examine the efficacy of TRIM35 knockdown in BC cell lines. B) Efficacy of TRIM35 knockdown in BC cell lines was detected by IB. C) Cell proliferation assay in BC cells with depletion of TRIM35. D) Measurement of apoptosis in cells with indicated treatments by flow cytometry. E) Quantification of flow cytometry in D.

MCF-7 cell lines upon TRIM35 overexpressing. To validate the findings, we then depleted TRIM35 in SKBR3 cell line, and similarly followed by IB to detect AKT signaling and apoptosis. Vice versa, we observed that depletion of TRIM35 significantly increased the level of phosphor AKT at S473 in SKBR3 cell line. In agreement, reduction of Bad but the increase of XIAP were observed in SKBR3 cell line upon TRIM35 depletion (Figure 4C). In addition, SKBR3 cells were transfected with both TRIM35 knockdown and overexpression lentiviruses. The results showed that the effects induced by TRIM35 depletion were rescued by TRIM35 overexpression (Figure 4C). Altogether, TRIM35 regulates the AKT signaling and apoptosis in $\mathrm{BC}$ cell lines.

TRIM35 negatively regulates PDK1 in BC cells. As reported, $\mathrm{PI} 3 \mathrm{~K}, \mathrm{PDK} 1$, and $\mathrm{PDK} 2$ are the negative regulators of AKT $[24,25]$, and TRIM35 was found to inactivate the AKT signaling pathway (Figure 4). Therefore, we assumed that TRIM 35 could bind to these negative regulators, due to its E3 ligase activity. As shown in Figure 5A, TRIM35 interacted with PDK1 only, suggesting that TRIM35 regulated 
the AKT signaling pathway through PDK1. We further confirmed the interaction between TRIM35 and PDK1 in SKBR3 cell line (Figure 5B). Next, we sought to investigate whether and how TRIM35 regulated PDK1 in BC cell lines. Firstly, we overexpressed TRIM35 in ZR7530 and MCF-7 cell lines, followed by IB to detect the level of PDK1. As shown in Figure 5C, overexpression of TRIM35 significantly reduced the protein level of PDK1 in ZR7530 and MCF-7 cell lines. To validate these findings, we then depleted TRIM35 in SKBR3 cell line and similarly followed by IB to detect the protein level of PDK1. Vice versa, we observed that depletion of TRIM35 significantly increased the protein level of PDK1 in SKBR3 cells.

TRIM35 enhances the ubiquitination of PDK1. Both transcriptional and post-transcriptional regulations could affect the protein levels of PDK1 upon TRIM35 manipulations. To identify the mechanisms contributing to the reduction of PDK1 upon TRIM35 overexpression, we firstly measured the mRNA levels of TRIM35 in BC cell lines with TRIM35 overexpression or depletion. As illustrated in
A



B
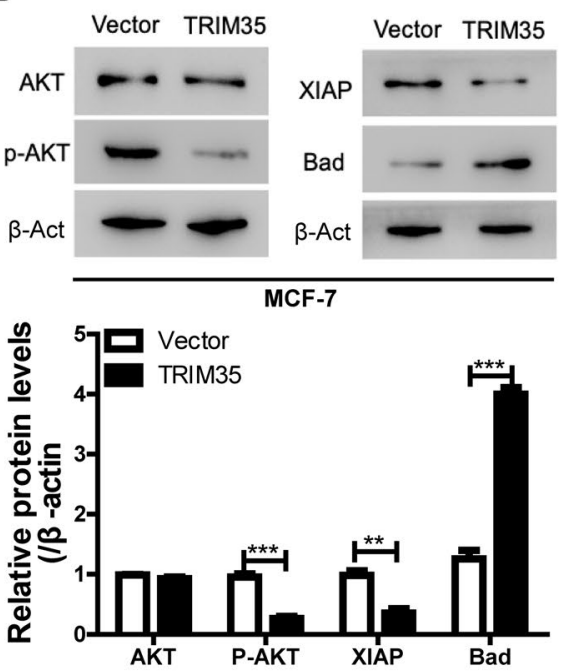


C


Figure 4. TRIM35 induces apoptosis through inactivating the AKT signaling. A) Gene set enrichment assay (GSEA) to examine the correlation between gene signatures and TRIM35. B) Cells overexpressing TRIM35 were harvested for IB. C) Cells with depletion of TRIM35 or TRIM35 overexpression were harvested for IB. ${ }^{*} \mathbf{p}<0.05$ vs. sh-NC; $\# \mathbf{p}<0.05$ vs. sh-TRIM35+TRIM35. 
A

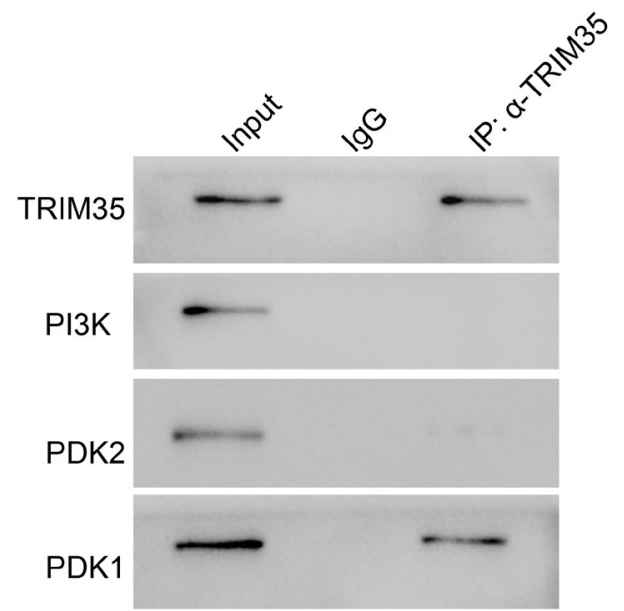

B



C
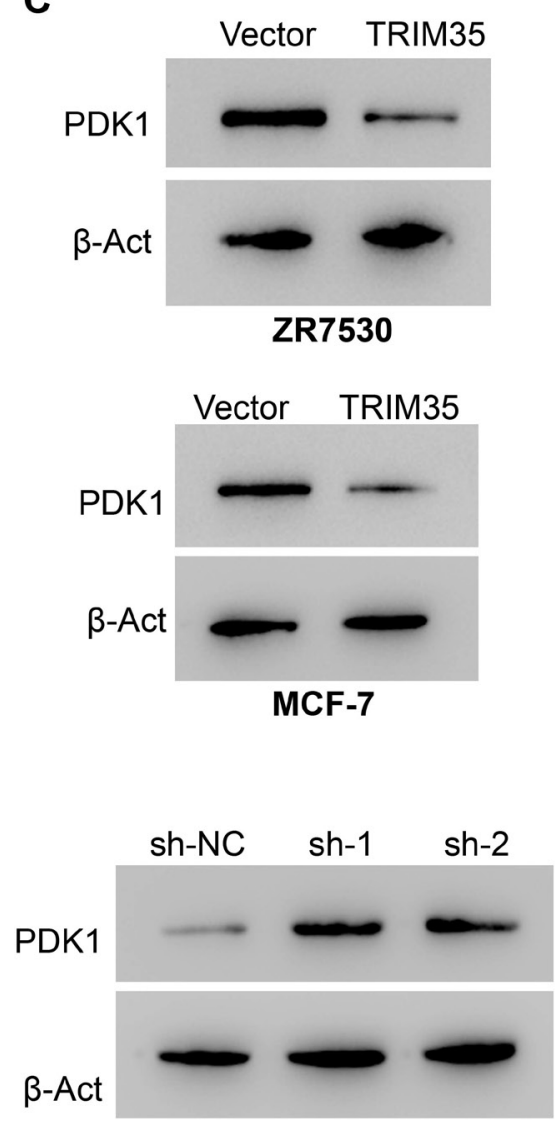

SKBR3

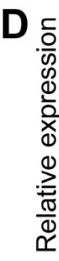
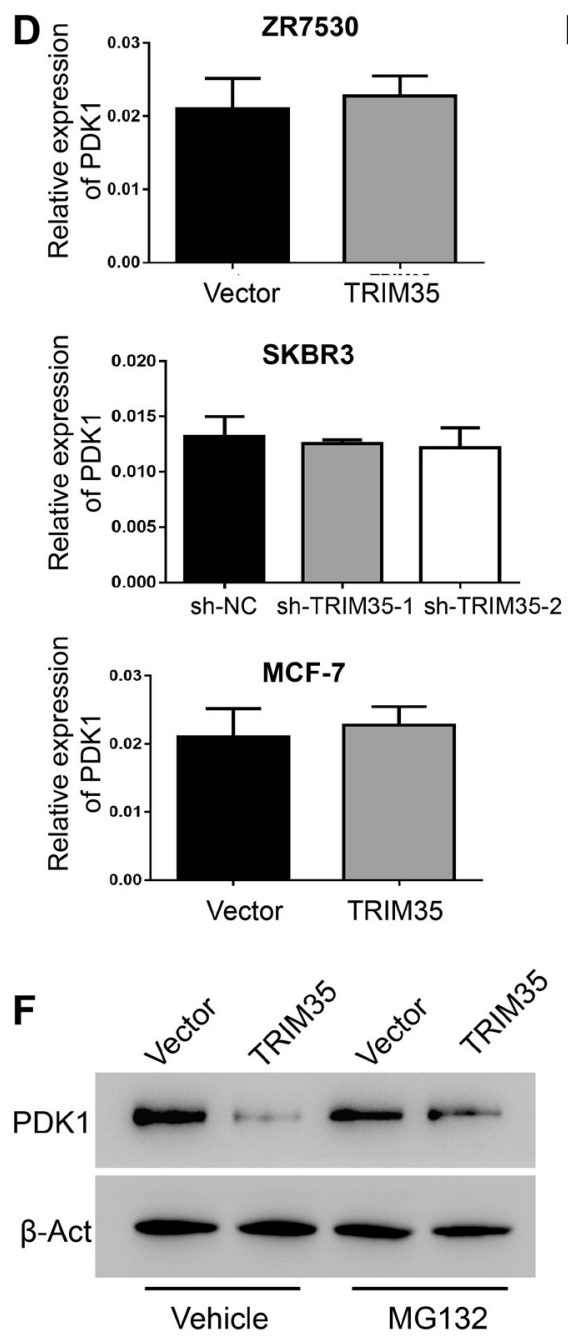

E
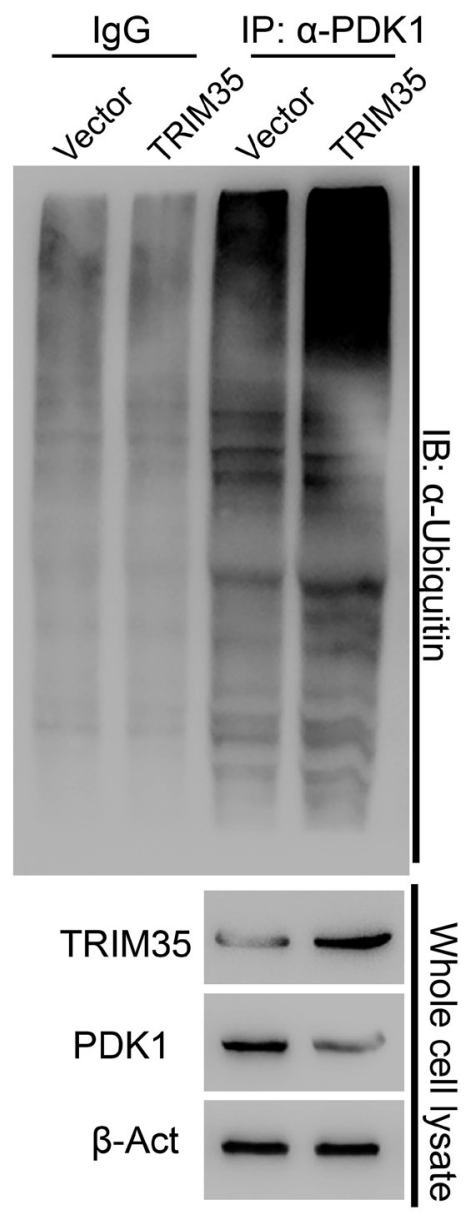

Figure 5. TRIM35 reduces the level of PDK1 by promoting its ubiquitination. A) Detecting interactions between TRIM35 and some negative regulators of AKT (PI3K, PDK1, and PDK2) by co-IP. B) Confirming the interaction between TRIM35 and PDK1 by co-IP. C) Cells with TRIM35 overexpression or depletion were harvested and followed by IB against PDK1. D) Cells with TRIM35 overexpression or depletion were harvested and followed by QPCR to measure transcription of PDK1. E) Cells overexpressing TRIM35 were treated with $10 \mathrm{mM}$ MG132 overnight, then harvested and used for IP against PDK1, and then followed by IB against ubiquitin. F) Cells with TRIM35 overexpression were treated with or without $10 \mathrm{mM}$ MG132 for overnight and followed by IB. G) Cells with overexpression of TRIM35 and TRIM35-mut and followed by IB. 
Figure 5D, no dramatic mRNA changes were observed in these cells upon TRIM35 manipulations. Next, we sought to investigate whether PDK1 was post-transcriptionally regulated. Considering that TRIM35 is a member of the RBCC family and possesses an E3 ligase activity, we hypothesized that TRIM35 might induce the degradation of PDK1 via its ubiquitination. Therefore, we then performed an immunoprecipitation assay in ZR7530 cell line to detect the ubiquitination level of PDK1 upon TRIM35 overexpression. In agreement, a higher level of ubiquitination of PDK1 was observed in cells with TRIM35 overexpression than that in cells transfected with vectors (Figure 5E). To further explore whether the reduction of PDK1 is proteasome pathwaydependent, we introduced MG132 to treat cells overexpressing TRIM35. As indicated, the reduction of PDK1 induced by TRIM35 overexpression can be attenuated by MG132 treatment, suggesting that TRIM35-induced reduction of PDK1 was proteasome pathway dependent (Figure $5 \mathrm{~F}$ ). As reported, the single C36S or C60S mutation was sufficient to abolish the E3 ligase activity of TRIM35, we made TRIM35-mut with both C36S and C60S mutation [26]. As shown in Figure 5G, TRIM35-mut can't decrease the PDK1 level. Taken together, TRIM35 decreases the protein level of PDK1 in BC cell lines through ubiquitination, resulting in proteasome-mediated degradation of PDK1.

The increase of cell proliferation and decrease of apoptosis induced by TRIM35 silencing could be alleviated by PDK1 depletion. Based on the finding described above, we then raised the hypothesis that TRIM35-induced cell apoptosis was PDK1 mediated. To test this notion, we depleted PDK1 in SKBR3 cell line. As illustrated in Figure $6 \mathrm{~A}, \mathrm{PDK} 1$ was effectively depleted in SKBR3 cells via shRNAs transfection. Similarly, an immunoblot assay also showed a significant reduction of PDK1 after transfection of shRNAs against PDK1 (Figure 6B). To study the effect of TRIM35 on the level of PDK1, we co-transfected SKBR3 cells with shRNAs against TRIM35 and/or PDK1. It was observed that depletion of TRIM35 could partially rescue the reduction of PDK1 induced by PDK1 knockdown (Figure 6C).

To further study the roles of PDK1 and TRIM35 in BC behaviors, we depleted PDK1 and TRIM35 in SKBR3 cell line, then subjected it to cell proliferation assay by measuring the absorbance at OD 450. As shown in Figure 6D, depletion of TRIM35 significantly stimulated cell proliferation in SKBR3 cell line $(\mathrm{p}<0.0001)$. On contrary, the depletion of PDK1 inhibited cell proliferation. Interestingly and expectedly, the knockdown of PDK1 can partially reverse the stimulation of cell growth induced by the depletion of TRIM35. These results were in accordance with the findings in Figure 6C. In addition, depletion of TRIM35 significantly decreased apoptosis in SKBR3 cell line which can be attenuated by depletion of PDK1 (Figures 6E, 6F). Mechanistically, depletion of TRIM35 significantly increased the level of phosphor AKT in SKBR3 cell line which can be attenuated by depletion of PDK1 (Figure 6G). Taken together, TRIM35-induced cell apoptosis was PDK1 mediated.

Enhanced expression of TRIM35 inhibits the growth of ZR7530-derived xenograft tumor. The findings in vitro suggested the role of TRIM35 on inhibiting cell growth, providing the possibilities for further studies in vivo. To this end, we constructed the ZR7530-derived xenograft tumor model by injecting ZR7530 cells subcutaneously. As shown in Figures 7A-C, overexpression of TRIM35 significantly suppressed tumor growth in vivo. To study the histological changes of the tumor with TRIM35 overexpression, we then performed $\mathrm{H} \& \mathrm{E}$ staining and observed more apoptotic niches in the tumors with TRIM35 overexpression (Figure 7D, red rectangle). In agreement, more TUNNEL staining was observed in TRIM35 overexpressing tumors, indicating more apoptosis persistence (Figure 7E). Mechanistically, overexpression of TRIM35 inactivated AKT signaling by decreasing the level of phosphor AKT in the tumors (Figure 7F). In summary, TRIM35 overexpressing induces apoptosis by inactivating AKT signaling resulting in tumor suppression in vivo.

\section{Discussion}

TRIM35 was documented as a protein with apoptotic function [15] and a candidate tumor suppressor [27]. In 2014, TRIM35 was identified as a novel tumor suppressor by using genome-wide single nucleotide polymorphism (SNP) 6.0 arrays in hepatocellular carcinoma [28] and TRIM35 could suppress the Warburg effect and tumorigenicity by interacting with pyruvate kinase isoform M2 in hepatocellular carcinoma [21].

Even the functions of TRIM35 in BC were not touched yet, these studies still provide the possibility for TRIM35 to be a tumor suppressor. As expected, we observed that the overexpression of TRIM35 significantly inhibited BC cell proliferation. Meanwhile, TRIM35 overexpression robustly promoted BC cell apoptosis as well (Figure 2). These data strongly suggested TRIM35 as a candidate for a tumor suppressor. Due to the complexity of the tumor cells, it's not surprising to observe the heterogenicity of BC cells. For example, TRIM35 was highly expressed in SKBR3 cell line but lowly expressed in MCF-7 and ZR7530 cell lines. The complexities imply the different roles of TRIM35 in BC. Herein, it is reasonable to raise the hypothesis that TRIM35 plays distinct roles in $\mathrm{BC}$ with different histological characteristics. This notion raises the need to further study the roles of TRIM35 in BC in the future. Since TRIM35 inhibited cell proliferation and promoted cell apoptosis, it is required to clarify which function of TRIM35 is dominant or TRIM35 inhibits cell proliferation by inducing cell apoptosis.

Next, we sought to dissect the mechanisms contributing to the phenotypes observed. GSEA was performed in human patients and identified the inactivation of AKT signaling (Figure 4A). This finding was recapitulated in vitro 
A

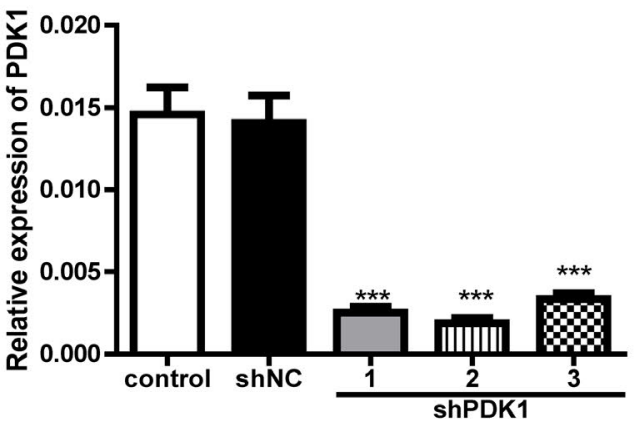

C


sh-NC




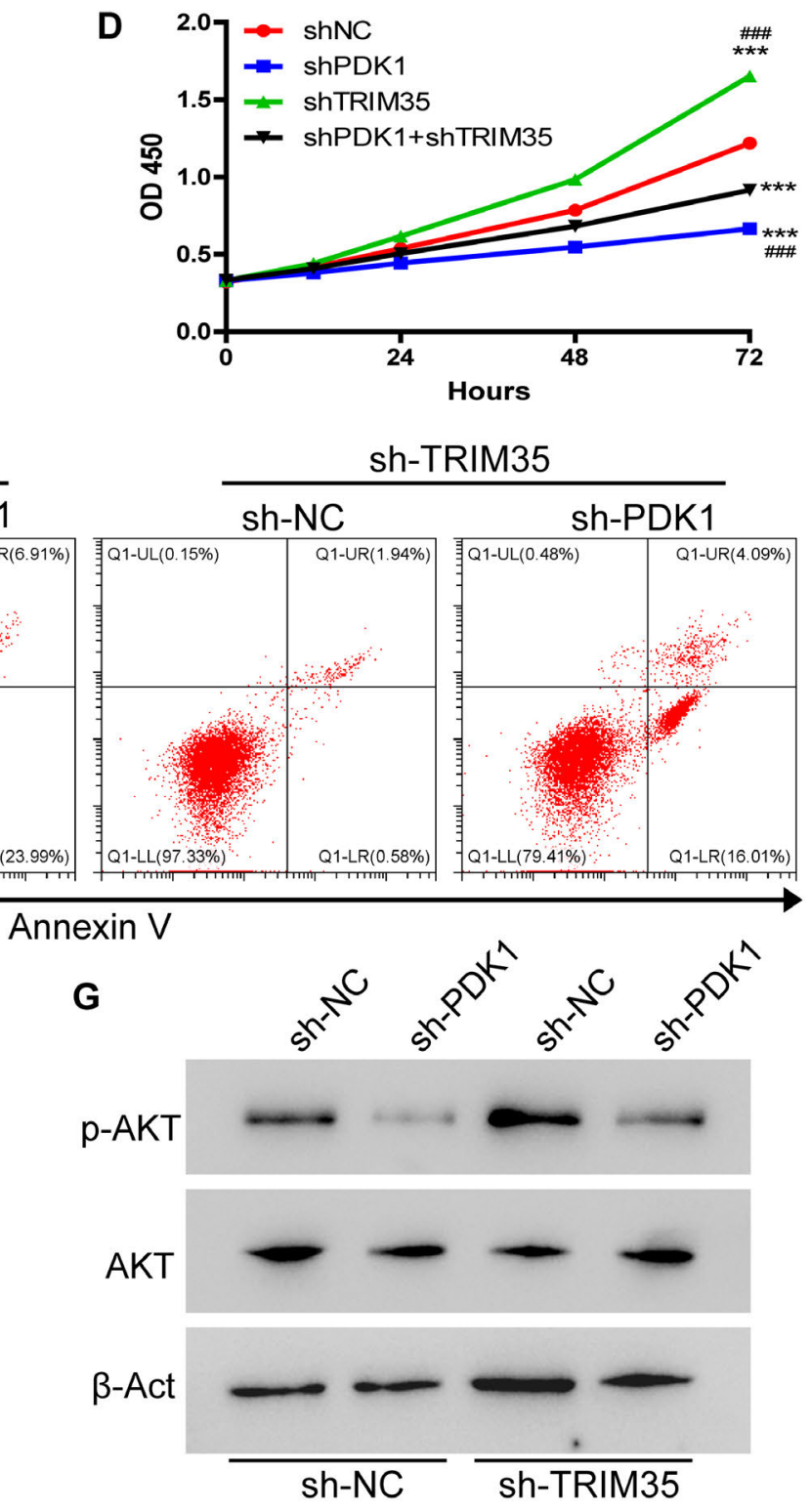

Figure 6. Depletion of TRIM35 promotes cell proliferation can be attenuated by PDK1 knockdown. A) qPCR to examine the efficacy of PDK1 knockdown in BC cell lines. B) Efficacy of PDK1 knockdown in BC cell lines was detected by IB. C) Cells received indicated treatments were harvested for IB. D) Cell proliferation assay in cells with indicated treatments. E) Measurement of apoptosis in cells with indicated treatments by flow cytometry. F) Quantification of flow cytometry in E. G, Cells with depletion of PDK1 were harvested for IB. 
A

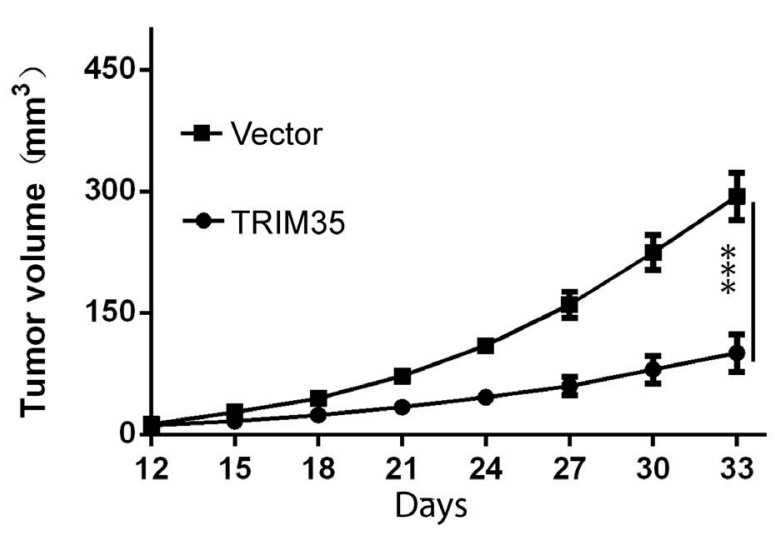

C

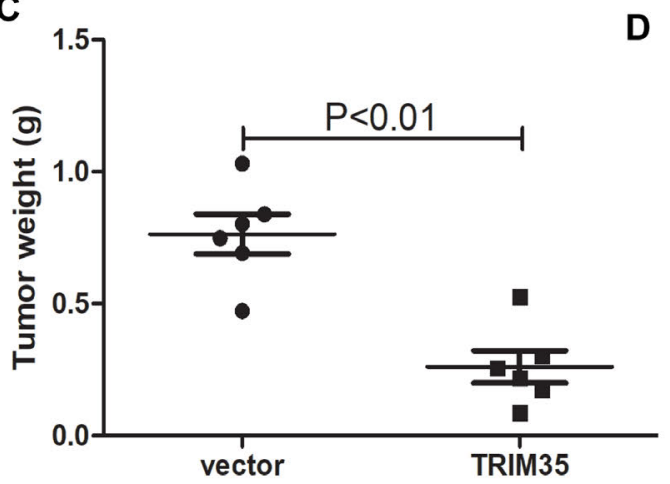

E


\#3

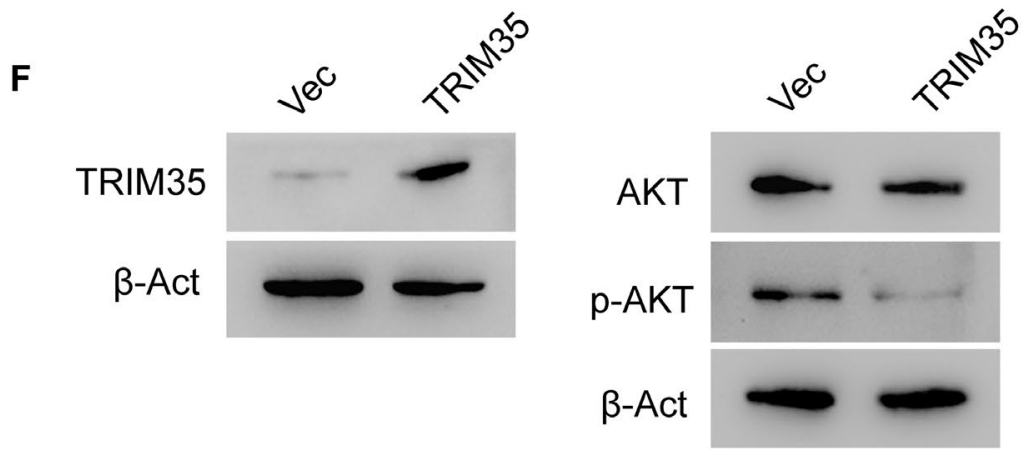

B

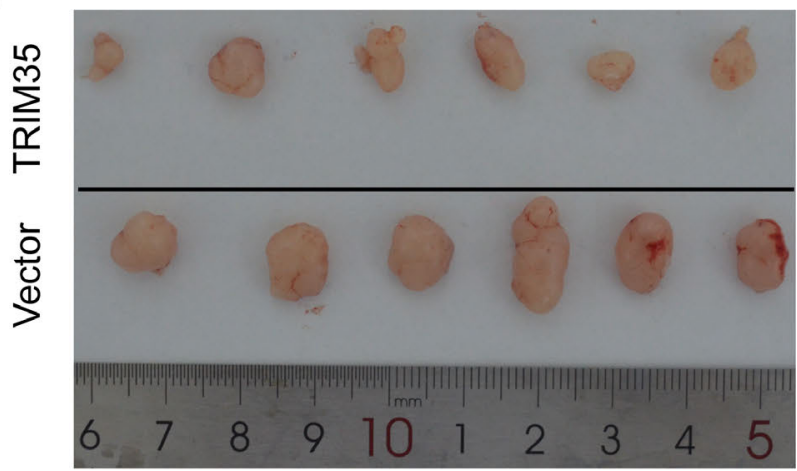

\#3 
as reduction of phosphor AKT was observed upon TRIM35 overexpression (Figure 4B). To be an E3 ligase, this finding raises the possibility that TRIM35 may regulate phosphorylation of AKT by targeting a potential kinase in BC. PDK1 and PDK2 have been established to phosphorylate AKT [29], implying they could be the substrates of TRIM35. It has been documented that PDK1 phosphorylates AKT at T308 and PDK2 may phosphorylate AKT at S473 [29]. It is reasonable to detect the changes of PDK1 and PDK2 upon TRIM35 overexpression (Figure 5A). Interestingly, PDK1 was regulated post-transcriptionally (Figures 5B, 5C). It's not surprising to observe more ubiquitination of PDK1 upon TRIM35 overexpression in considering it is a candidate of E3 ligase. However, what is the role of PDK2 in the activating AKT signaling is not clear yet. Another concern is that the AKT signaling pathway should be fully investigated even we showed strong clues for that.

In addition to the role of TRIM35, PDK1 exerted the opposite effects against TRIM35. Similarly, since depletion of PDK1 inhibited cell proliferation and promoted cell apoptosis (Figure 6), it is required to clarify which function of PDK1 is dominant or depletion of PDK1 inhibits cell proliferation by inducing cell apoptosis. Despite the depletion of PDK1 was performed in the study, other methods, such as PDK1 and TRIM35 inhibitors should be used to validate our findings because we only paid attention to the protein level of PDK1 and TRIM35 in the present study, however, the enzyme activity of PDK1 and TRIM35 is still not understood.

We also validated our findings with the ZR7530 cellderived xenograft tumor model and observed similar results. However, it is not great to recapitulate the characteristics of human BC. To this end, the patient-derived xenograft (PDX) tumor model should be applied in the future to validate the roles of TRIM35 and PDK1 further. In addition, orthotopic xenograft models are required to mimic the growth of breast tumors in vivo. In parallel, potential targeted inhibitors can be introduced to test the possibilities to translate our findings into clinical trials.

In conclusion, TRIM35 plays a tumor suppressor role in $\mathrm{BC}$ by inactivating the AKT signaling pathway through increased ubiquitination of PDK1.

Acknowledgments: This study was funded by the Scientific research project of the Shanghai Health Committee (201940505).

\section{References}

[1] ALKABBAN F M, FERGUSON T. Breast Cancer, Stat Pearls. StatPearls [Internet]. Treasure Island (FL): StatPearls Publishing; 2021.

[2] ALTUNDAG K. Detailed information about chemotherapy in breast control arm might affect cognitive sequelae compared with endocrine therapy patients. Breast Cancer Res Treat 2018; 169: 203. https://doi.org/10.1007/s10549-018$4672-7$
[3] HANDFORTH C, D'ORONZO S, COLEMAN R, BROWN J. Cancer Treatment and Bone Health. Calcif Tissue Int 2018; 102: 251-264. https://doi.org/10.1007/s00223-017-0369-x

[4] TANG X, SHI L, XIE N, LIU Z, QIAN M et al. SIRT7 antagonizes TGF-beta signaling and inhibits breast cancer metastasis. Nat Commun 2017; 8: 318. https://doi.org/10.1038/ s41467-017-00396-9

[5] WU Y, TRAN T, DWABE S, SARKISSYAN M, KIM J et al. A83-01 inhibits TGF-beta-induced upregulation of Wnt3 and epithelial to mesenchymal transition in HER2-overexpressing breast cancer cells. Breast Cancer Res Treat 2017; 163: 449-460. https://doi.org/10.1007/s10549-017-4211-y

[6] SHAN S, LV Q, ZHAO Y, LIU C, SUN Y et al. Wnt/betacatenin pathway is required for epithelial to mesenchymal transition in CXCL12 over expressed breast cancer cells. Int J Clin Exp Pathol 2015; 8: 12357-12367.

[7] AGRAWAL AK, PIELKA E, LIPINSKI A, JELEN M, KIELAN W et al. Clinical validation of nuclear factor kappa B expression in invasive breast cancer. Tumour Biol 2018; 40: 1010428317750929. https://doi. org/10.1177/1010428317750929

[8] LEE K J, YOO JW, KIM YK, CHOI JH, HA TY et al. Advanced glycation end products promote triple negative breast cancer cells via ERK and NF-kappaB pathway. Biochem Biophys Res Commun 2018; 495: 2195-2201. https:// doi.org/10.1016/j.bbrc.2017.11.182

[9] BAEK SH, KO JH, LEE JH, KIM C, LEE H et al. Ginkgolic Acid Inhibits Invasion and Migration and TGF-beta-Induced EMT of Lung Cancer Cells Through PI3K/Akt/mTOR Inactivation. J Cell Physiol 2017; 232: 346-354. https://doi. org/10.1002/jcp. 25426

[10] GRILLE S J, BELLACOSA A, UPSON J, KLEIN-SZANTO A J, VAN ROY F et al. The protein kinase Akt induces epithelial mesenchymal transition and promotes enhanced motility and invasiveness of squamous cell carcinoma lines. Cancer Res 2003; 63: 2172-2178.

[11] KUANG W, DENG Q, DENG C, LI W, SHU S et al. Hepatocyte growth factor induces breast cancer cell invasion via the PI3K/Akt and p38 MAPK signaling pathways to up-regulate the expression of COX2. Am J Transl Res 2017; 9: 3816-3826.

[12] YANG W, SCHWARTZ GN, MAROTTI JD, CHEN V, TRAPHAGEN NA et al. Estrogen receptor alpha drives mTORC1 inhibitor-induced feedback activation of PI3K/ AKT in ER+ breast cancer. Oncotarget 2018; 9: 8810-8822. https://doi.org/10.18632/oncotarget.24256

[13] CHIA S, GANDHI S, JOY AA, EDWARDS S, GORR M et al. Novel agents and associated toxicities of inhibitors of the pi3k/Akt/mtor pathway for the treatment of breast cancer. Curr Oncol 2015; 22: 33-48. https://doi.org/10.3747/ co. 22.2393

[14] ALVAREZ RH, BECHARA RI, NAUGHTON MJ, ADACHI JA, REUBEN JM. Emerging Perspectives on mTOR Inhibitor-Associated Pneumonitis in Breast Cancer. Oncologist 2018; 23: 660-669. https://doi.org/10.1634/theoncologist.2017-0343

[15] KIMURA F, SUZU S, NAKAMURA Y, NAKATA Y, YAMADA $M$ et al. Cloning and characterization of a novel RINGB-box-coiled-coil protein with apoptotic function. J Biol Chem 2003; 278: 25046-25054. https://doi.org/10.1074/jbc. M303438200 
[16] CIECHANOVER A. The unravelling of the ubiquitin system. Nat Rev Mol Cell Biol 2015; 16: 322-324. https://doi. org/10.1038/nrm3982

[17] LEE JH, LIU R, LI J, ZHANG C, WANG Y et al. Stabilization of phosphofructokinase 1 platelet isoform by AKT promotes tumorigenesis. Nat Commun 2017; 8: 949. https://doi. org/10.1038/s41467-017-00906-9

[18] TANG SL, GAO YL, WEN-ZHONG H. Knockdown of TRIM37 suppresses the proliferation, migration and invasion of glioma cells through the inactivation of PI3K/Akt signaling pathway. Biomed Pharmacother 2018; 99: 59-64. https://doi.org/10.1016/j.biopha.2018.01.054:

[19] WANG Y, YAN S, YANG B, WANG Y, ZHOU H et al. TRIM35 negatively regulates TLR7- and TLR9-mediated type I interferon production by targeting IRF7. FEBS Lett 2015; 589: 1322-1330. https://doi.org/10.1016/j.febslet.2015.04.019

[20] SONG L, ZHANG W, CHANG Z, PAN Y, ZONG H et al. miR-4417 Targets Tripartite Motif-Containing 35 (TRIM35) and Regulates Pyruvate Kinase Muscle 2 (PKM2) Phosphorylation to Promote Proliferation and Suppress Apoptosis in Hepatocellular Carcinoma Cells. Med Sci Monit 2017; 23: 1741-1750. https://doi.org/10.12659/msm.900296

[21] CHEN Z, WANG Z, GUO W, ZHANG Z, ZHAO F et al. TRIM35 Interacts with pyruvate kinase isoform M2 to suppress the Warburg effect and tumorigenicity in hepatocellular carcinoma. Oncogene 2015; 34: 3946-3956. https://doi. org/10.1038/onc.2014.325

[22] BOS P D, ZHANG XH, NADAL C, SHU W, GOMIS RR et al. Genes that mediate breast cancer metastasis to the brain. Nature 2009; 459: 1005-1009. https://doi.org/10.1038/nature08021
[23] WEI L, WEN JY, CHEN J, MA XK, WU DH et al. Oncogenic ADAM28 induces gemcitabine resistance and predicts a poor prognosis in pancreatic cancer. World J Gastroenterol 2019; 25: 5590-5603. https://doi.org/10.3748/wjg.v25. i37.5590:

[24] LIN HJ, HSIEH FC, SONG H, LIN J. Elevated phosphorylation and activation of PDK-1/AKT pathway in human breast cancer. Br J Cancer 2005; 93: 1372-1381. https://doi. org/10.1038/sj.bjc.6602862:

[25] YANG L, QIAO G, YING H, ZHANG J, YIN F. TCR-induced Akt serine 473 phosphorylation is regulated by protein kinase C-alpha. Biochem Biophys Res Commun 2010; 400: 16-20. https://doi.org/10.1016/j.bbrc.2010.07.126

[26] SUN N, JIANG L, YE M, WANG Y, WANG G et al. TRIM35 mediates protection against influenza infection by activating TRAF3 and degrading viral PB2. Prot Cell 2020; 11: 894914. https://doi.org/10.1007/s13238-020-00734-6

[27] LALONDE JP, LIM R, INGLEY E, TILBROOK PA, THOMPSON MJ et al. HLS5, a novel RBCC [ring finger, B box, coiled-coil] family member isolated from a hemopoietic lineage switch, is a candidate tumor suppressor. J Biol Chem 2004; 279: 8181-8189. https://doi.org/10.1074/jbc. M306751200:

[28] JIA D, WEI L, GUO W, ZHA R, BAO M et al. Genome-wide copy number analyses identified novel cancer genes in hepatocellular carcinoma. Hepatology 2011; 54: 1227-1236. https://doi.org/10.1002/hep.24495

[29] DONG LQ, LIU F. PDK2: the missing piece in the receptor tyrosine kinase signaling pathway puzzle. Am J Physiol Endocrinol Metab 2005; 289: E187-196. https://doi.org/10.1152/ ajpendo.00011.2005 